\title{
Shock Waves in the Newman-Penrose Formalism
}

\author{
A. Papapetrou \\ Institute of Relativity Studies, Boston University, Boston and Institut Henri Poincaré, Paris
}

Received May 23, 1973

\begin{abstract}
Gravitational shock waves of order $n=2$ are considered and their description in radiation coordinates is discussed. It is found that in such coordinates there exist necessarily (trivial) discontınuities of first derivatives of $g_{\mu \nu}$. The structure of the propagation relation for this form of the shock wave is derived and the discontinuities of the NewmanPenrose field variables are determined.
\end{abstract}

\section{Discontinuities of the Newman-Penrose Field Variables}

In the study of shock waves it has been found that besides the essential discontinuities of the field variables there are also trivial discontinuities which can be eliminated by appropriate transformations. In the usual treatment of shock waves it is assumed that these discontinuities have been eliminated.

In certain cases however this elimination may conflict with some other demand we put on the description of the field. The case we shall consider here in detail concerns a gravitational field described in radiation (Bondi) coordinates. More specifically we shall use the Newman-Penrose formalism [1] and we shall consider a shock wave which is essentially of order $n=2$ and propagates on the null-surface $\Sigma$ determined by the equation $x^{0}=0$. We shall show that, because of the use of radiation coordinates, there will necessarily be present discontinuities of certain first derivatives of the metric.

Since the shock wave is (essentially) of order $n=2$, we shall have discontinuities of at least some of the scalars $\Psi^{A}$ determining the Weyl tensor $^{1}$. A priori we shall assume that certain first derivatives of the tetrad components $U, X^{\kappa}, \omega$ and $\xi^{\kappa}(\kappa=2,3)$ may also be discontinuous. Similarly we may have discontinuities of the rotation coefficients $\varrho, \sigma, \alpha$, $\beta, \gamma, \lambda, \mu$ and $\nu$, as these coefficients contain first derivatives of the tetrad components. A systematic use of the Newman-Penrose equations will allow us to determine the derivatives of the different field quantities which are really discontinuous.

\footnotetext{
1 We are using the notation introduced in [1]
} 
We firstly consider the coefficient $\varrho$ defined by

$$
\varrho=l_{\mu ; v} m^{\mu} \bar{m}^{v} \text {. }
$$

In the frame used by Newman and Penrose we have:

$$
\left.\begin{array}{rlrl}
l_{\mu} & =(1,0,0,0), & & l^{\mu}=(0,1,0,0) ; \\
m^{\mu} & =\left(0, \omega, \xi^{2}, \xi^{3}\right) ; & n^{\mu} & =\left(1, U, X^{2}, X^{3}\right) .
\end{array}\right\}
$$

Therefore $l_{\mu, v}=0$ and consequently

$$
\varrho=-\Gamma_{\mu \nu}^{\alpha} l_{\alpha} m^{\mu} \bar{m}^{v}=-\frac{1}{2}\left(g_{1 \mu, v}+g_{1 v, \mu}-g_{\mu v, 1}\right) m^{\mu} \bar{m}^{v} .
$$

The first two terms vanish because $g_{1 \mu}=\delta_{\mu 0}$. The last term is continuous across $\Sigma$ since $x^{1} \equiv r$ is a coordinate on $\Sigma$. Therefore

$$
[\varrho] \equiv \varrho_{+}-\varrho_{-}=0 \text {. }
$$

A reasoning of exactly the same type shows that the coefficients $\sigma$ and $\tau=\bar{\alpha}+\beta$ are also continuous:

$$
[\sigma]=[\tau]=0 .
$$

We now consider the coefficient $\mu$,

$$
\mu=-n_{\alpha ; \beta} \bar{m}^{\alpha} m^{\beta} \text {. }
$$

According to (1) we have $\alpha, \beta \neq 0$ and consequently

$$
\begin{gathered}
{\left[n_{\alpha, \beta}\right]=\left[g_{\gamma \alpha, \beta}\right]=\left[g_{\beta \gamma, \alpha}\right]=0 ;} \\
{[\mu]=-\frac{1}{2}\left[g_{\alpha \beta, \gamma}\right] n^{\gamma} \bar{m}^{\alpha} m^{\beta} .}
\end{gathered}
$$

The discontinuity $\left[g_{\alpha \beta, \gamma}\right]$ being trivial, we shall have

and consequently:

$$
\begin{aligned}
{\left[g_{\alpha \beta, \gamma}\right] } & =\beta_{\alpha \beta} l_{\gamma}, \\
\beta_{\alpha \beta} & =B_{\alpha} l_{\beta}+B_{\beta} l_{\alpha}
\end{aligned}
$$

$$
[\mu]=0 \text {. }
$$

A reasoning of exactly the same type leads to the results:

$$
[\lambda]=[\alpha]=[\beta]=0 .
$$

In order to continue we have to use the other sets of the NewmanPenrose equations. We shall not write these equations here in detail; we shall refer to them by their numbers in [2]. We start with Eq. (11d). Integrating both sides of this equation over the interval $-\varepsilon \leqq x^{0} \leqq \varepsilon$, $\varepsilon \rightarrow 0$, we find:

$$
\left[\Psi^{3}\right]=0
$$


otherwise we should have $\Psi^{4} \sim \delta\left(x^{0}\right)$, contrary to our assumption (shock wave of essential order $n=2)$. Similarly we find from Eq. (11c), (11b) and (11 a):

$$
\left[\Psi^{2}\right]=\left[\Psi^{1}\right]=\left[\Psi^{0}\right]=0 .
$$

From Eq. (9j) we get at once:

$$
[D \gamma]=D[\gamma]=0 .
$$

Therefore $[\gamma]=f(\theta, \varphi)$. Since $\gamma \rightarrow 0$ for $r \rightarrow \infty$, we must have

$$
[\gamma]=0 \text {. }
$$

By a similar reasoning we derive from $(9 \mathrm{~m})$ :

$$
[v]=0 \text {. }
$$

Turning again to Eq. (11 b) we find, using the results we have obtained until now:

$$
\left[\dot{\Psi}^{1}\right]=0
$$

the dot meaning derivative with respect to $x^{0}$. Similarly we get from (11a):

$$
\left[\dot{\Psi}^{0}\right]=0 \text {. }
$$

In a similar way we get from $(10 \mathrm{~d})$ and $(10 \mathrm{a})$ :

$$
[\dot{\omega}]=\left[\dot{\xi}^{\kappa}\right]=0,
$$

and from Eq. (10e) and (10i) to (10m):

$$
[\dot{\lambda}]=[\dot{\mu}]=[\dot{\beta}]=[\dot{\sigma}]=[\dot{Q}]=[\dot{\alpha}]=0 \text {. }
$$

Finally we find from $(9 \mathrm{c})$, after differentiating it with respect to $x^{0}$ :

$$
D\left[\dot{X}^{\kappa}\right]=0 \text {. }
$$

Since $X^{\kappa} \rightarrow 0$ when $r \rightarrow \infty$ we must have

$$
\left[\dot{X}^{\kappa}\right]=0 \text {. }
$$

Recapitulating we find that the following quantities will be discontinuous:

$$
\left.\begin{array}{l}
\dot{U} ; \ddot{X}^{\kappa}, \ddot{\omega}, \ddot{\xi}^{\kappa} ; \\
\dot{\gamma}, \dot{\lambda}, \dot{v} ; \ddot{\varrho}, \ddot{\sigma}, \ddot{\alpha}, \ddot{\beta}, \ddot{\mu} ; \\
\Psi^{4} ; \dot{\Psi}^{3}, \dot{\Psi}^{2} ; \ddot{\Psi}^{1}, \ddot{\Psi}^{0} .
\end{array}\right\}
$$

The formula

$$
g^{\lambda \mu}=l^{\lambda} n^{\mu}+l^{\mu} n^{\lambda}-\left(m^{\lambda} \bar{m}^{\mu}+m^{\mu} \bar{m}^{\lambda}\right)
$$


shows that the discontinuities of $g^{\lambda \mu}{ }_{, v}$ will depend on $[\dot{U}]$. Starting from the relation

with $\beta_{\lambda \mu}$ of the form (4),

$$
\left[g^{\lambda \mu}, v\right]=-\beta^{\lambda \mu} l_{v}
$$

we find from (15):

$$
\beta^{\lambda \mu}=B^{\lambda} l^{\mu}+B^{\mu} l^{\lambda}
$$

$$
B^{1}=0, \quad B^{2}=-[\dot{U}], \quad B^{3}=B^{4}=0 .
$$

Hence the vector $B^{\lambda}$ satisfies the relation

$$
B^{\lambda} l_{\lambda}=0 \text {. }
$$

Using again the Newman-Penrose equations we can express the discontinuities of all quantities appearing in the Table (A) in terms of $\left[\Psi^{4}\right]$. We give as example the following relations, resulting from the Eq. (11d), (11 c), (10e) and the time derivatives of Eqs. (9j) and (9d):

$$
\begin{aligned}
& \left.\begin{array}{l}
{\left[\dot{\Psi}^{3}\right]=\delta\left[\Psi^{4}\right]+(4 \beta-\tau)\left[\Psi^{4}\right],} \\
{\left[\dot{\Psi}^{2}\right]=\sigma\left[\Psi^{4}\right]}
\end{array}\right\} \\
& {[\dot{i}]=-\left[\Psi^{4}\right] \text {; }} \\
& \partial_{r}[\dot{\gamma}]=\sigma\left[\Psi^{4}\right], \\
& \left.\partial_{r}[\dot{U}]=-[\dot{\gamma}]-[\dot{\bar{\gamma}}] .\right\}
\end{aligned}
$$

From $(9 \mathrm{q})$ we get an equation for $\left[\Psi^{4}\right]$ :

$$
\partial_{r}\left[\Psi^{4}\right]=\varrho\left[\Psi^{4}\right] \text {. }
$$

Eq. (21) is identical with the propagation relation derived by the classical treatment of a gravitational shock wave, as we shall show in the following.

\section{The Classical Treatment of the Problem}

In the classical description of shock waves the problem we have been considering here is characterised as follows. There are discontinuities of the first derivatives of $g_{\lambda \mu}$, but they are trivial, the first essential discontinuities being those of the second derivatives. Id est formally we have a shock wave of order $n=1$, but essentially of order $n=2$.

Shock waves of order $n=1$, propagating on the hypersurface $z=0$ (in the present case $z=x^{0}$ ), have been discussed in detail [3]. The discussion is based on the formula

$$
\stackrel{+}{g}_{\mu v}=\bar{g}_{\mu v}+z \beta_{\mu v}+\frac{z^{2}}{2} \gamma_{\mu v}+\frac{z^{3}}{6} \varepsilon_{\mu v}+\cdots,
$$


$\stackrel{+}{g}_{\mu \nu}$ being the metric for $z>0 ; \bar{g}_{\mu \nu}$ is the metric for $z<0$ and its continuation in the region $z>0$ having continuous derivatives up to the order 3. A direct calculation leads to the formulae:

$$
2\left(\stackrel{+}{\Gamma}_{\mu \nu}^{\alpha}-\bar{\Gamma}_{\mu v}^{\alpha}\right)=\Lambda_{\mu \nu}^{\alpha}+z \Lambda_{\mu \nu}^{\prime \alpha}+\frac{z^{2}}{2} \Lambda_{\mu \nu}^{\prime \alpha}+\cdots ;
$$

$$
\begin{aligned}
& \Lambda_{\mu \nu}^{\alpha}=\beta^{\alpha}{ }_{\mu} l_{v}+\beta^{\alpha}{ }_{v} l_{\mu}-l^{\alpha} \beta_{\mu \nu},
\end{aligned}
$$

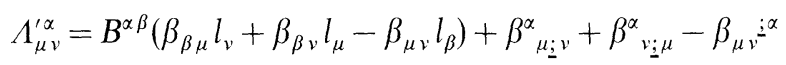

$$
\begin{aligned}
& +\gamma^{\alpha}{ }_{\mu} l_{v}+\gamma^{\alpha}{ }_{v} l_{\mu}-l^{\alpha} \gamma_{\mu v} \text {, } \\
& A_{\mu \nu}^{\prime \prime \alpha}=\Gamma^{\alpha \beta}\left(\beta_{\beta \mu} l_{v}+\beta_{\beta v} l_{\mu}-\beta_{\mu \nu} l_{\beta}\right)+2 B^{\alpha \beta}\left(\beta_{\beta \mu ; \nu}+\beta_{\beta v i j \mu}-\beta_{\mu v ; \beta}+\gamma_{\beta \mu} l_{v}\right.
\end{aligned}
$$

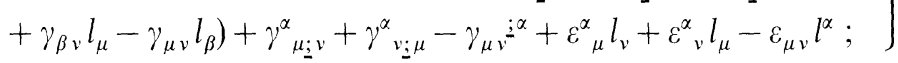

$$
\begin{aligned}
& B^{\mu v}=-\beta^{\mu v}, \quad \Gamma^{\mu v}=-\gamma^{\mu v}+2 \beta_{\alpha}^{\mu} \beta^{\alpha v} ; \\
& 2\left(\stackrel{+}{R}_{\mu \nu}-\bar{R}_{\mu \nu}\right)=\left\{-\Lambda_{\mu v ; \alpha}^{\alpha}+\Lambda_{\mu \alpha ; v}^{\alpha}-\Lambda_{\mu \nu}^{\prime \alpha} l_{\alpha}+\Lambda_{\mu \alpha}^{\prime \alpha} l_{v}+\frac{1}{2} \Lambda_{\beta \mu}^{\alpha} \Lambda_{\alpha v}^{\beta}-\frac{1}{2} \Lambda_{\beta \alpha}^{\alpha} \Lambda_{\mu v}^{\beta}\right\} \\
& +z\left\{-\Lambda_{\mu v \underline{i} \alpha}^{\prime \alpha}+\Lambda_{\mu \alpha_{i} v}^{\prime \alpha}-\Lambda_{\mu v}^{\prime \prime \alpha} l_{\alpha}+\Lambda_{\mu \alpha}^{\prime \prime \alpha} l_{v}+\frac{1}{2} \Lambda_{\beta \mu}^{\alpha} \Lambda_{\alpha v}^{\prime \beta}\right. \\
& \left.+\frac{1}{2} \Lambda_{\beta \nu}^{\alpha} \Lambda_{\alpha \mu}^{\prime \beta}-\frac{1}{2} \Lambda_{\beta \alpha}^{\alpha} \Lambda_{\mu \nu}^{\prime \beta}-\frac{1}{2} \Lambda_{\beta \alpha}^{\prime \alpha} \Lambda_{\mu \nu}^{\beta}\right\}+O\left(z^{2}\right) .
\end{aligned}
$$

In these formulae indices are raised with the metric $\bar{g}^{\mu v}$ and the symbol ; denotes covariant derivation with respect to $\bar{\Gamma}_{\mu \nu}^{\alpha}$.

Eq. (23) shows that $\Gamma_{\mu \nu}^{\alpha}$ contains a term of the form $\Lambda_{\mu \nu}^{\alpha} S(z), S(z)$ being the step-function. Therefore $R_{\mu \nu}$ will contain a term proportional to $\frac{d}{d z}-S(z)=\delta(z)$ :

$$
R_{\mu \nu}=\delta(z)\left\{-\Lambda_{\mu \nu}^{\alpha} l_{\alpha}+\Lambda_{\mu \alpha}^{\alpha} l_{v}\right\}+\cdots .
$$

The omitted part is finite, but discontinuous at $z=0$ and its discontinuity is given by (26). In order to have the vacuum field equations satisfied also on the surface $z=0$ we have to put the coefficient of $\delta(z)$ in (27) equal to zero:

$$
\Lambda_{\mu \nu}^{\alpha} l_{\alpha}-\Lambda_{\mu \nu}^{\alpha} l_{v}=0
$$

This is the local condition which leads, in the case of a shock wave of (essentially) the order $n=1$, to the conclusions:

$$
\begin{gathered}
l^{\alpha} l_{\alpha}=0 ; \\
\beta_{\mu v}=a\left(m_{\mu} m_{v}+\bar{m}_{\mu} \bar{m}_{v}\right)+\frac{b}{i}\left(m_{\mu} m_{v}-\bar{m}_{\mu} \bar{m}_{v}\right)+B_{\mu} l_{v}+B_{v} l_{\mu} .
\end{gathered}
$$


The next condition we have to demand is the vanishing of the term in (26) which is independent of $z$ :

$$
-\Lambda_{\mu v \underline{\alpha} \alpha}^{\alpha}+\Lambda_{\mu \alpha ; v}^{\alpha}-\Lambda_{\mu v}^{\alpha} l_{\alpha}+\Lambda_{\mu \alpha}^{\prime \alpha} l_{v}+\frac{1}{2}\left(\Lambda_{\beta \mu}^{\alpha} \Lambda_{\alpha v}^{\beta}-\Lambda_{\beta \alpha}^{\alpha} \Lambda_{\mu v}^{\beta}\right)=0 .
$$

The detailed discussion of this equation leads to the propagation condition:

$$
2 a_{, \mu} l^{\mu}+a l_{: \mu}^{\mu}=0=2 b_{, \mu} l^{\mu}+b{l^{\mu}}_{; \mu} .
$$

These relations are the real and the imaginary part of the equation

$$
2\left(\beta_{\mu \nu} m^{\mu} m^{v}\right)_{, \alpha} l^{\alpha}+\beta_{\mu v} m^{\mu} m^{v} l_{; \alpha}^{\alpha}=0
$$

which is obtained when we multiply (31) by $m^{\mu} m^{v}$. What remains in (31) is then a relation between $\gamma_{\mu}=\frac{1}{2} \gamma_{\alpha}^{\alpha} l_{\mu}-\gamma_{\mu \alpha} l^{\alpha}$ and $\beta_{\mu \nu}$.

The case we are considering here is characterized by $a=b=0$. Eq. (28) is then satisfied trivially. It is now Eq. (31) which will play the role of the local condition ${ }^{2}$. The propagation condition will in this case be derived from the equation expressing the vanishing of the coefficient of $z$ in (26), which is equivalent to the demand

$$
\left[\frac{d}{d z} R_{\mu v}\right]=0
$$

This is a rather lengthy equation and we shall not give it here in detail. The propagation condition is again obtained when we multiply this equation by $m^{\mu} m^{\nu}$. The final result is:

$$
2\left\{\left(\gamma_{\mu \nu}-B_{\mu_{i} v}\right) m^{\mu} m^{\nu}\right\}_{, \lambda} l^{\lambda}+\left(\gamma_{\mu \nu}-B_{\mu \underline{i}, v}\right) m^{\mu} m^{v} l_{; \lambda}^{\lambda}=0 .
$$

This is the generalisation of the propagation condition for a shock wave of essential order 2, in the presence of trivial discontinuities of first derivatives of $g_{\lambda \mu}$.

We now consider the Weyl tensor $C_{\lambda \mu v \varrho}$ which in the vacuum case is identical with the Riemann tensor:

$$
C_{\lambda \mu \nu \varrho}=R_{\lambda \mu \nu \varrho} .
$$

One verifies directly that because of the condition (28) $R_{\lambda \mu v e}$ does not contain a term proportional to $\delta(z)$. We shall calculate the discontinuity

\footnotetext{
2 Derıving the relation (29) from this condition is rather complicated. It will be simples to consider Eq. (29) as valid on the basis of the discussion of the shock wave in a frame in which the trivial discontinuties have been eliminated. This is permitted because Eq. (29) is an invariant relation.
} 
$\left[R_{\lambda \mu \nu}\right]$ starting from the relation:

$2 R_{\lambda \mu v \varrho}=g_{\lambda \varrho, \mu v}+g_{\mu v, \lambda \varrho}-g_{\lambda v, \mu \varrho}-g_{\mu \varrho, \lambda v}+2\left(\Gamma_{\mu v}^{\alpha} \Gamma_{\alpha, \lambda \varrho}-\Gamma_{\mu \varrho}^{\alpha} \Gamma_{\alpha, \lambda v}\right)$.

From (22) we find:

$$
\begin{aligned}
{\left[g_{\lambda \mu, v}\right] } & =\beta_{\lambda \mu} l_{v}, \\
{\left[g_{\lambda \mu, v \varrho}\right] } & =\beta_{\lambda \mu, v} l_{\varrho}+\beta_{\lambda \mu, \varrho} l_{v}+\beta_{\lambda \mu} l_{v, \varrho}+\gamma_{\lambda \mu} l_{v} l_{\varrho} .
\end{aligned}
$$

Using also the relation:

$$
[\Gamma \Delta] \equiv \stackrel{+}{\Gamma} \Delta-\bar{\Gamma} \Delta=\bar{\Gamma}[\Delta]+[\Gamma] \Delta+[\Gamma][\Delta]
$$

we find after some elementary calculations:

$$
\left.\begin{array}{rl}
2\left[R_{\mu v \varrho \sigma}\right]= & \beta_{\mu \sigma ; v} l_{\varrho}+\beta_{\mu \sigma ; \varrho} l_{v}+\beta_{\mu \sigma} l_{v \underline{\Sigma} \varrho}+\gamma_{\mu \sigma} l_{v} l_{\varrho} \\
& +\beta_{v \varrho ; \mu} l_{\sigma}+\beta_{v \varrho ; \sigma} l_{\mu}+\beta_{v \varrho} l_{\mu ; \sigma}+\gamma_{v \varrho} l_{\mu} l_{\sigma} \\
& -\beta_{\mu \varrho \vdots v} l_{\sigma}-\beta_{\mu \varrho \vdots \sigma} l_{v}-\beta_{\mu \varrho} l_{v ; \sigma}-\gamma_{\mu \varrho} l_{v} l_{\sigma} \\
& -\beta_{v \sigma ; \mu} l_{\varrho}-\beta_{v \sigma \vdots \varrho} l_{\mu}-\beta_{v \sigma} l_{\mu ; \varrho}-\gamma_{v \sigma} l_{\mu} l_{\varrho} .
\end{array}\right\}
$$

The scalar $\Psi^{4}$ is defined by the relation $(12 n)$ of [2]:

Therefore:

$$
\Psi^{4}=R_{\mu \nu \varrho \sigma} \bar{m}^{\mu} n^{v} n^{\varrho} \bar{m}^{\sigma} .
$$

$$
2\left[\Psi^{4}\right]=2\left[R_{\mu \nu \varrho \sigma}\right] \bar{m}^{\mu} n^{v} n^{\varrho} \bar{m}^{\sigma} .
$$

Multiplying (36) by $\bar{m}^{\mu} n^{v} n^{\varrho} \bar{m}^{\sigma}$ we find:

$$
2\left[\Psi^{4}\right]=\left\{2 \beta_{\mu \sigma_{i} v} n^{v}+\gamma_{\mu \sigma}+\beta_{v \varrho} n^{v} n^{\varrho} l_{\mu ; \sigma \sigma}-2 \beta_{\mu \varrho ; \sigma} n^{\varrho}-2 \beta_{\mu \varrho} n^{\varrho} l_{v_{\Sigma} \sigma} n^{v}\right\} \bar{m}^{\mu} \bar{m}^{\sigma} \text {. }
$$

Introducing in this relation the expression (4) for $\beta_{\mu \sigma}$ we find finally:

$$
2\left[\Psi^{4}\right]=\left(\gamma_{\mu \sigma}-2 B_{\mu ; \sigma}\right) \bar{m}^{\mu} \bar{m}^{\sigma} .
$$

This result shows that the propagation relation (34) can be written in the more intuitive form:

$$
2\left[\Psi^{4}\right]_{, \alpha} l^{\alpha}+\left[\Psi^{4}\right] l_{; \alpha}^{\alpha}=0
$$

which is equivalent to (21) because of

and the form (1) of $l^{\alpha}$.

$$
\varrho=-\frac{1}{2} l_{; \alpha}^{\alpha}
$$

The author wishes to thank Dr. J. Stachel for valuable discussions. 


\title{
References
}

1. Newman, E., Penrose, R.: J. Math. Phys. 3, 566 (1962)

2. Newman,E.T., Unti, T. W.J.: J. Math. Phys. 3, 891 (1962)

3. Papapetrou,A., Treder, H.: Math. Nachrichten 20, 53 (1959)

\author{
A. Papapetrou \\ Institut Henri Poincaré \\ Lab. de Physique Théorique \\ 11, rue Pierre et Marie Curie \\ F-75231 Paris, Cedex 05, France
}

